\title{
Observing the First Stars, One Star at a Time
}

\author{
Abraham Loeb \\ Astronomy Department, Harvard University, 60 Garden Street, Cambridge, \\ MA 02138, USA; \\ aloeb@cfa.harvard.edu
}

Summary. Gamma-Ray Bursts (GRBs) are believed to originate in compact remnants (black holes or neutron stars) of massive stars. Their high luminosities make them detectable out to the edge of the visible universe. We describe the many advantages of GRB afterglows relative to quasars as probes of the intergalactic medium during the epoch of reionization. The Swift satellite, planned for launch by the end of 2004 , will likely open a new era in observations of the high redshift universe.

\section{Stellar Explosions at the Edge of the Visible Universe}

Gamma-Ray Bursts (GRBs) are believed to originate in compact remnants (black holes or neutron stars) of massive stars. Their high luminosities make them detectable out to the edge of the visible universe $[13,24]$. GRBs offer the opportunity to detect the most distant (and hence earliest ${ }^{1}$ ) population of massive stars, one star at a time. In the hierarchical assembly process of halos which are dominated by cold dark matter, the first galaxies should have had lower masses (and lower stellar luminosities) than their low-redshift counterparts. Consequently, the characteristic luminosity of galaxies or quasars is expected to decline with increasing redshift. GRB afterglows, which already produce a peak flux comparable to that of quasars or starburst galaxies at $z \sim 1-2$, are therefore expected to outshine any competing source at the highest redshifts, when the first dwarf galaxies have formed in the universe.

Preliminary polarization data from the Wilkinson Microwave Background Probe ( $W M A P$ ) indicates an optical depth to electron scattering of $\sim 17 \pm 4 \%$ after cosmological recombination [30]. This implies that the first stars must have formed at a redshift $z \sim 20[11,12,14,29,35,36]$ and reionized a substantial fraction of the intergalactic hydrogen around that time. Early reionization can be achieved with plausible star formation parameters in the standard $\Lambda \mathrm{CDM}$ cosmology; in fact, the required optical depth can be achieved in a variety of very different ionization histories (since WMAP places only an integral constraint on these histories [20]). One would like to probe the

\footnotetext{
${ }^{1}$ Observational cosmology resembles archaeology. By probing deeper into the universe, one reveals layers of it that are more ancient (due to the finite speed of light).
} 
full history of reionization in order to disentangle the properties and formation history of the stars that are responsible for it. GRB afterglows offer the opportunity to detect stars as well as to probe the ionization state [5] and metal enrichment level [17] of the intervening intergalactic medium (IGM).

GRBs, the electromagnetically-brightest explosions in the universe, should be detectable out to redshifts $z>10[13,24]$. High-redshift GRBs can be easily identified through infrared photometry, based on the Ly $\alpha$ break induced by absorption of their spectrum at wavelengths below $1.216 \mu \mathrm{m}[(1+z) / 10]$. Follow-up spectroscopy of high-redshift candidates can then be performed on a 10-meter-class telescope. There are four main advantages of GRBs relative to traditional cosmic sources such as quasars:

- The GRB afterglow flux at a given observed time lag after the $\gamma$-ray trigger is not expected to fade significantly with increasing redshift, since higher redshifts translate to earlier times in the source frame, during which the afterglow is intrinsically brighter [13]. For standard afterglow lightcurves and spectra, the increase in the luminosity distance with redshift is compensated by this "cosmological time-stretching" effect.

- As already mentioned, in the standard $\Lambda \mathrm{CDM}$ cosmology, galaxies form hierarchically, starting from small masses and increasing their average mass with cosmic time. Hence, the characteristic mass of quasar black holes and the total stellar mass of a galaxy were smaller at higher redshifts, making these sources intrinsically fainter [34]. However, GRBs are believed to originate from a stellar mass progenitor and so the intrinsic luminosity of their engine should not depend on the mass of their host galaxy. GRB afterglows are therefore expected to outshine their host galaxies by a factor that gets larger with increasing redshift.

- Since the progenitors of GRBs are believed to be stellar, they likely originate in the most common star-forming galaxies at a given redshift rather than in the most massive host galaxies, as is the case for bright quasars [5]. Low-mass host galaxies induce only a weak ionization effect on the surrounding IGM and do not greatly perturb the Hubble flow around them. Hence, the Ly $\alpha$ damping wing should be closer to the idealized unperturbed IGM case [27] and its detailed spectral shape should be easier to interpret. Note also that unlike the case of a quasar, a GRB afterglow can itself ionize at most $\sim 4 \times 10^{4} E_{51} M_{\odot}$ of hydrogen if its UV energy is $E_{51}$ in units of $10^{51} \mathrm{ergs}$ (based on the available number of ionizing photons), and so it should have a negligible cosmic effect on the surrounding IGM.

- GRB afterglows have smooth (broken power-law) continuum spectra unlike quasars which show strong spectral features (such as broad emission lines or the so-called "blue bump") that complicate the extraction of IGM absorption features. In particular, the continuum extrapolation into the Ly $\alpha$ damping wing (the Gunn-Peterson [19] absorption trough) during the epoch of reionization is much more straightforward for the smooth 
UV spectra of GRB afterglows than for quasars with an underlying broad Ly $\alpha$ emission line $[4,5]$.

Although the nature of the central engine that powers the relativistic jets of GRBs is unknown, recent evidence indicates that GRBs trace the formation of massive stars $[6,7,23,32,33]$, and in particular that long-duration GRBs are associated with Type Ib/c supernovae [31]. Since the first stars in the universe are predicted to be predominantly massive $[1,8]$, their death might give rise to large numbers of GRBs at high redshifts.

\section{GRB Afterglows at High Redshifts}

\subsection{Motivation}

In difference from quasars of comparable brightness, GRB afterglows are short-lived and release $\sim 10$ orders of magnitude less energy into the surrounding IGM. Beyond the scale of their host galaxy, they have a negligible effect on their cosmological environment ${ }^{2}$. Consequently, they make ideal probes of the IGM during the reionization epoch. Their rest-frame UV spectra can be used to probe the ionization state of the IGM through the spectral shape of the Gunn-Peterson [19] (Ly $\alpha$ ) absorption trough, or its metal enrichment history through the intersection of enriched bubbles of supernova ejecta from early galaxies[17]. Afterglows that are unusually bright ( $>10 \mathrm{mJy})$ at radio frequencies should also show a detectable forest of $21 \mathrm{~cm}$ absorption lines due to enhanced HI column densities in sheets, filaments, and collapsed mini-halos within the IGM $[10,16]$.

Another advantage of GRB afterglows is that once they fade away, one may search for their host galaxies. Hence, GRBs may serve as signposts of the earliest dwarf galaxies that are otherwise too faint or rare on their own for a dedicated search to find them. Detection of metal absorption lines from the host galaxy in the afterglow spectrum, offers an unusual opportunity to study the physical conditions (temperature, metallicity, ionization state, and kinematics) in the interstellar medium of these high-redshift galaxies.

\subsection{Afterglow Flux at the Ly $\alpha$ Wavelength}

Figure 1 shows the expected spectral flux from GRB afterglows as a function of observed time after the GRB trigger for a sequence of redshifts (assuming typical model parameters [5]). The flux is calculated at the rest-frame Ly $\alpha$ wavelength, where intergalactic HI should produce the Gunn-Peterson [19] trough prior to reionization. As already mentioned, the flux does not decline

\footnotetext{
${ }^{2}$ Note, however, that feedback from a single GRB or supernova on the gas confined within early dwarf galaxies could be dramatic, since the binding energy of most galaxies at $z>10$ is lower than $10^{51}$ ergs $[3,25]$.
} 


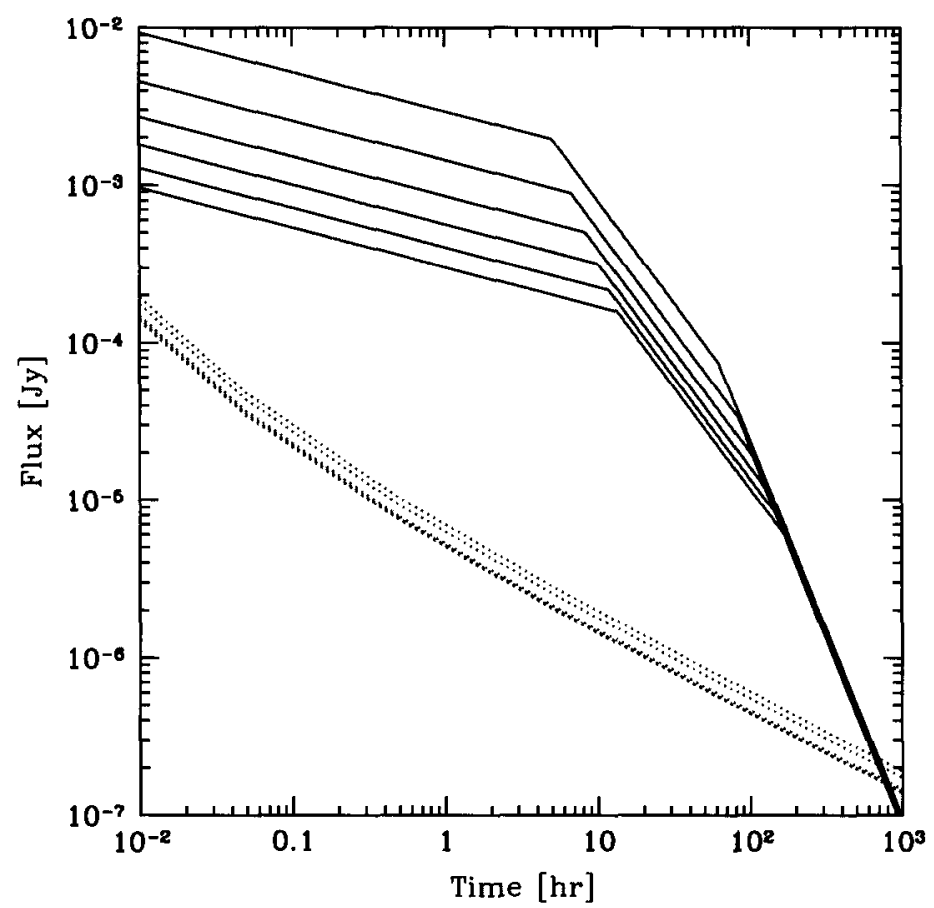

Fig. 1. GRB afterglow flux as a function of time since the $\gamma$-ray trigger in the observer frame (taken from [5]). The flux (solid curves) is calculated at the redshifted Ly $\alpha$ wavelength. The dotted curves show the planned detection threshold for the James Webb Space Telescope (JWST), assuming a spectral resolution $R=5000$ with the near infrared spectrometer, a signal to noise ratio of 5 per spectral resolution element, and an exposure time equal to $20 \%$ of the time since the GRB explosion (see http://www.ngst.stsci.edu/nms/main/). Each set of curves shows a sequence of redshifts, namely $z=5,7,9,11,13$, and 15 , respectively, from top to bottom.

dramatically with increasing redshift since cosmic time stretching counteracts the luminosity distance increase.

The expected spectral shape of the Gunn-Peterson trough prior to the final overlap phase of HII regions in the IGM, is shown in Fig. 2. If GRBs trace the typical sites of star formation, then most of them should be hosted by dwarf galaxies at high redshifts. Low-mass hosts would perturb only weakly the surrounding IGM (radiatively through their ionizing flux, gravitationally [through cosmological infall, or hydrodynamically through their winds), in difference from the massive host galaxies of the brightest quasars at the same epoch $[4,5]$. Consequently, the spectral shape of the Ly $\alpha$ trough (which is proportional to $e^{-\tau(\lambda)}$, where $\tau(\lambda)$ is the optical depth as a function of wavelength) is simpler to interpret for GRB afterglows than it is for quasars. The optical depth as a function of wavelength in the Ly $\alpha$ damping wing is 


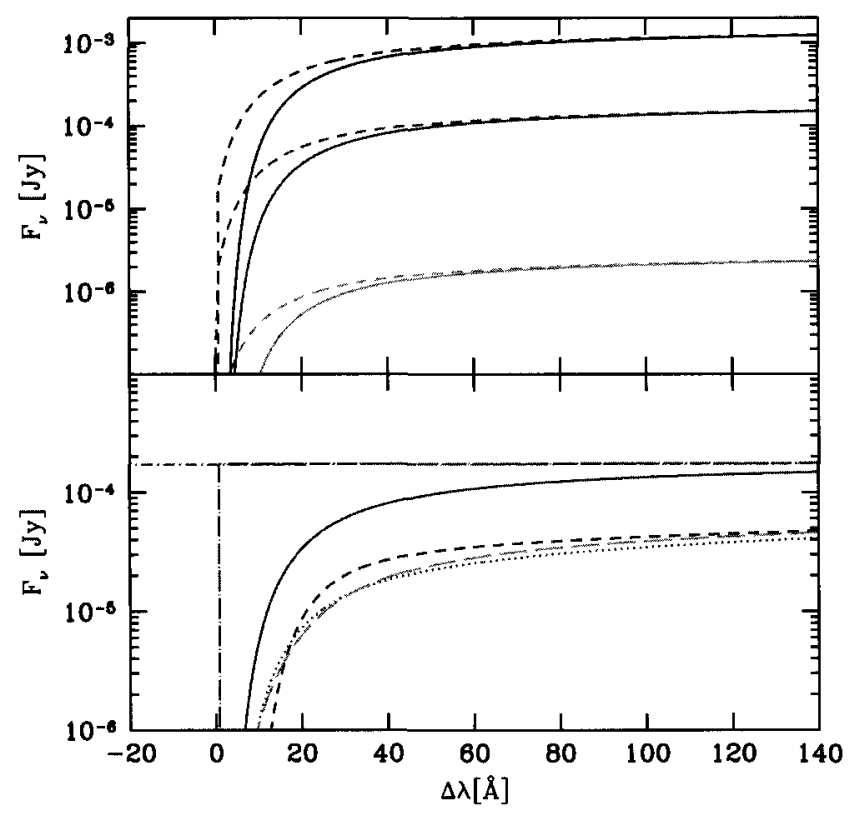

Fig. 2. Expected spectral shape of the Ly $\alpha$ absorption trough due to intergalactic absorption in GRB afterglows (taken from [5]). The spectrum is presented in terms of the flux density $F_{\nu}$ versus relative observed wavelength $\Delta \lambda$, for a source redshift $z=7$ (assumed to be prior to the final reionization phase) and the typical halo mass $M=4 \times 10^{8} M_{\odot}$ expected for GRB host galaxies that cool via atomic transitions. Top panel: Two examples for the predicted spectrum including IGM HI absorption (both resonant and damping wing), for host galaxies with: (i) an age $t_{S}=10^{7}$ yr, a UV escape fraction $f_{\mathrm{esc}}=10 \%$ and a Scalo initial mass function (IMF) in solid curves, or (ii) $t_{S}=10^{8} \mathrm{yr}, f_{\text {esc }}=90 \%$ and massive $\left(>100 M_{\odot}\right)$ Pop III stars in dashed curves. The observed time after the $\gamma$-ray trigger is one hour, one day, and ten days, from top to bottom, respectively. Bottom panel: Predicted spectra one day after a GRB for a host galaxy with $t_{S}=10^{7} \mathrm{yr}, f_{\text {esc }}=10 \%$ and a Scalo IMF. Shown is the unabsorbed GRB afterglow (dot-short dashed curve), the afterglow with resonant IGM absorption only (dot-long dashed curve), and the afterglow with full (resonant and damping wing) IGM absorption (solid curve). Also shown, with 1.7 magnitudes of extinction, are the afterglow with full IGM absorption (dotted curve), and attempts to reproduce this profile with a damped Ly $\alpha$ absorption system in the host galaxy (dashed curves). (Note, however, that damped absorption of this type could be suppressed by the ionizing effect of the afterglow UV radiation on the surrounding interstellar medium of its host galaxy [28].)

linearly proportional to the mean neutral fraction of the IGM, $x_{\mathrm{HI}}$, since its amplitude is normalized by the optical depth at the Ly $\alpha$ resonance, $\sim 6.75 \times$ $10^{5} x_{\mathrm{HI}}[(1+z) / 10]^{3 / 2}[3,25,27]$. 


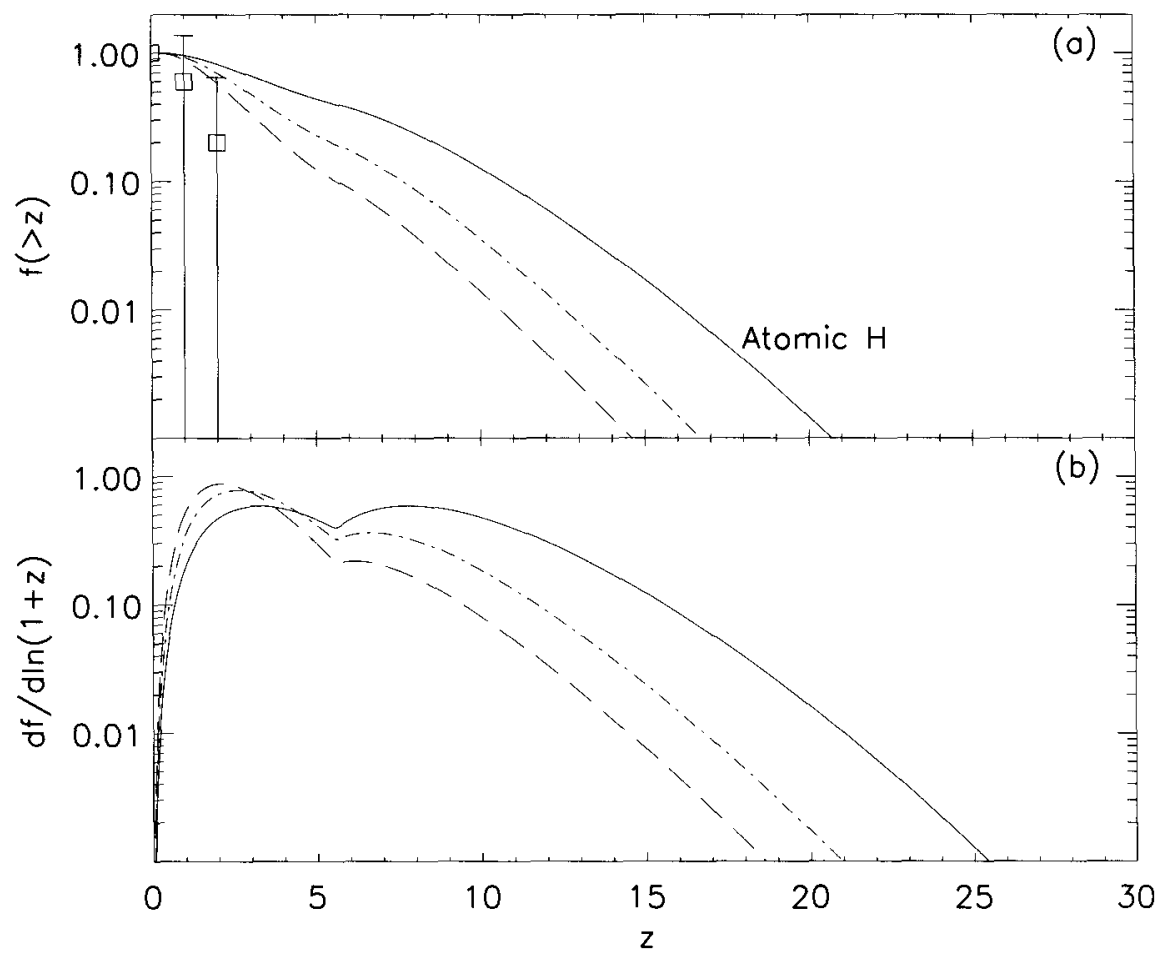

Fig. 3. Redshift distribution of all GRBs as compared to that measured by fluxlimited surveys (taken from [9]). (a) Fraction of bursts that originate at a redshift higher than $z$ vs. $z$. The data points reflect $\sim 20$ observed redshifts. (b) Fraction of bursts per logarithmic interval of $(1+z)$ vs. $z$. Solid lines: All GRBs for star formation through atomic line cooling. Dot-dashed lines: Expected distribution for Swift. Long-dashed lines: Expected distribution for BATSE. Note that the curves for the two flux-limited surveys are very uncertain due to the poorly-determined GRB luminosity function. Nevertheless, GRB number counts could provide a rough measure of the cosmic formation rate of massive stars at high redshifts.

\subsection{Statistics}

The upcoming Swift satellite (see http://swift.gsfc.nasa.gov/), planned for launch by the end of 2003, is expected to detect about a hundred GRBs per year. Fig. 3 shows the expected redshift distribution of all GRBs (solid lines), under the assumption that the GRB rate is simply proportional to the star formation rate [9]. The long-dashed and the dot-dashed lines show the same rate, but taking into account the limited sensitivity of the BATSE and Swift detectors, respectively. This calculation implies that about a quarter of all GRBs detected by Swift should originate at $z>5$. This estimate is rather uncertain because of the poorly determined GRB luminosity function [9]. 
In principle, the rate of high-redshift GRBs may be significantly suppressed if the early massive stars fail to launch a relativistic outflow. This is possible, since metal-free stars may experience negligible mass loss before exploding as a supernova. They would then retain their massive hydrogen envelope, and any relativistic jet might be quenched before escaping the star. However, localized metal enrichment is expected to occur rapidly (on a timescale much shorter than the age of the then-young universe) due to starbursts in the first galaxies and so even the second generation of star formation could occur in an interstellar medium with a significant metal content, resulting in massive stars that resemble more closely the counterparts of low-redshift GRB progenitors.

We note that the known population of optically-dark GRBs [23] is most likely associated with dust-obscured GRBs at low redshifts, although a small fraction of these bursts could be optically-dark due to Ly $\alpha$ absorption by the IGM at high-redshift.

\subsection{Progenitors}

In the previous section, we have assumed for simplicity a constant efficiency of forming GRBs per unit mass of stars. This simplifying assumption may either overestimate or underestimate the frequency of GRBs. Metal-free stars are thought to be massive $[1,8]$ and their extended envelopes may suppress central jets within them (which may be produced through the collapse of their core to a spinning black hole). On the other hand, low-metallicity stars are expected to have weak winds with little angular momentum loss during their evolution, and so they may preferentially yield rotating central configurations that produce GRB jets after core collapse $[18,22]$. The basic question: "Should GRBs be common in the first generation of metal-free stars?" remains open for further study.

\section{Swift: A Challenge for Infrared Follow-Ups}

A small fraction $(\sim 10-25 \%)$ of the GRB afterglows detected by Swift are expected to originate at redshifts $z>5$. This subset of afterglows can be selected photometrically using a small telescope, based on the Ly $\alpha$ break at a wavelength of $1.216 \mu \mathrm{m}[(1+z) / 10]$, caused by intergalactic HI absorption. The challenge in the upcoming years would be to follow-up on these candidates spectroscopically, using a large (10-meter class) telescope. A highresolution spectrum can then be used to trace the ionization state (see Fig. 2 ) and metal enrichment state of the gas along the line-of-sight. For example, Fig. 4 illustrates the expected number of intersections of enriched bubbles of supernova ejecta from early galaxies, as a function of the equivalent width of the metal absorption lines they produce (see [17] for details). Measurement of this statistic for different metals can be used to constrain the initial mass 


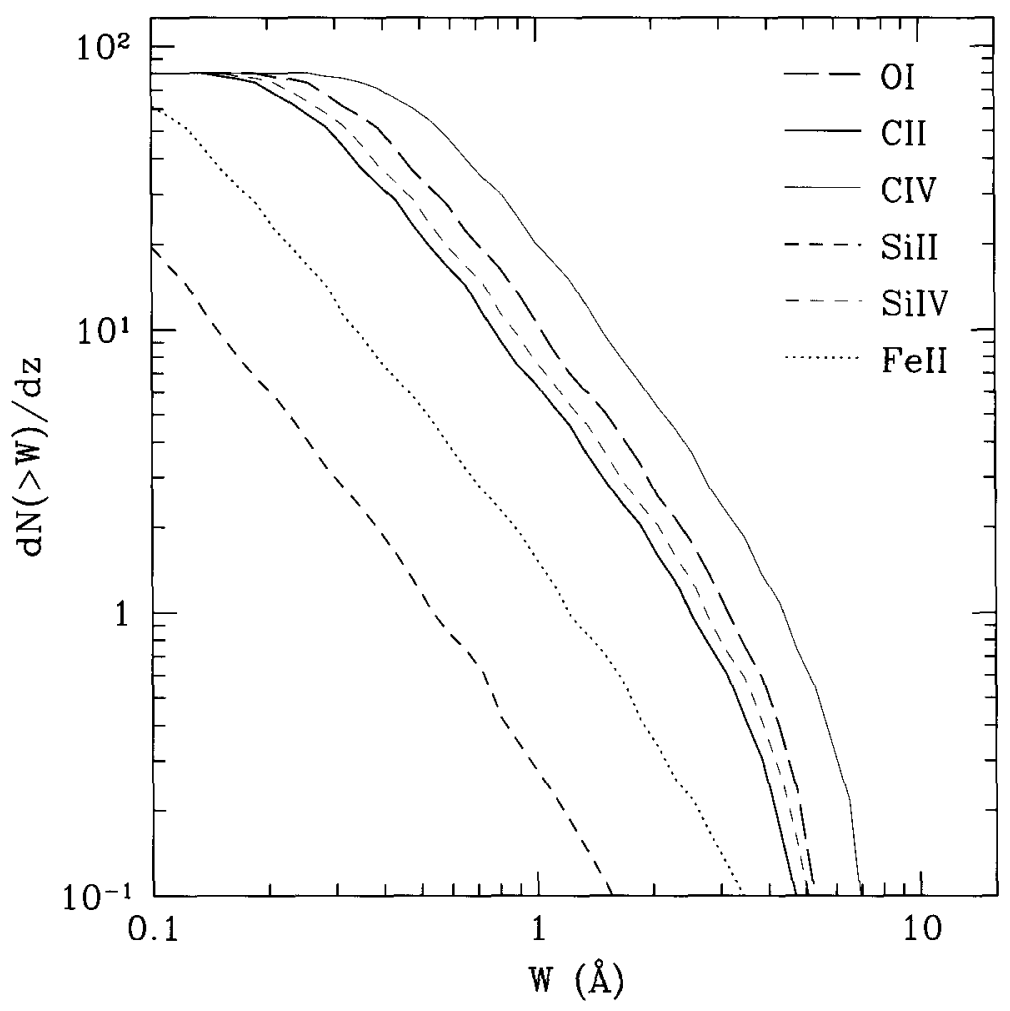

Fig. 4. Predicted number of line-of-sight intersections of metal-enriched bubbles around the first galaxies per redshift interval at a redshift $z=8$ (taken from [17]). This statistic was calculated as a function of the equivalent width threshold of the metal absorption lines produced by these bubbles, for typical parameter choices of the theoretical model (see [17] for details). Thick solid, long-dashed, short-dashed, and dotted curves are for C II, O I, Si II, and Fe II metal lines, respectively. Thin solid and short-dashed curves are for C IV and Si IV, respectively.

function (through element abundances) and the mechanical energy output (through bubble sizes) of the first stars, as well as the significance of molecular $\mathrm{H}_{2}$ cooling in forming stars within the first mini-halos (through the number density of small bubbles).

Based on the arguments mentioned in $\S 1$, GRB afterglows are likely to revolutionize observational cosmology and replace traditional sources like quasars, as probes of the IGM at $z>5$. We caution, however, that this is a theoretical expectation (the highest redshift GRB detected so far [2] is at $z \sim 4.5$ ) and as such it is subject to uncertainties about the production of relativistic outflows by the first generation of stars.

However, note that even if GRB outflows are quenched in the massive envelopes of low-metallicity stars, one would expect them to appear at high 
redshifts. This follows from the fact that metallicity obtains a large range of possible values (in different spatial regions) at any slice of cosmic time. For example, the broad emission lines of all known quasars show evidence for high metallicity gas $[15,21]$, and so it is clear that there are pockets of highly enriched gas near the centers of massive galaxies at $z>6$, where star formation reached an advanced stage and GRB production should be as likely as it is in the local universe. The cores of the most massive galaxies at $z>6$ simply predate the characteristic evolution of the rest of the universe at a much lower redshift, because these cores reside in unusually over dense cosmological environments [26]. Such environments are likely to produce GRB progenitors in the same way that less over dense cosmological regions produce them at a lower redshift. Swift has the potential to educate us whether GRBs are produced only within these massive galaxies or in the more common sites of star formation at early cosmic times, namely the low-metallicity dwarf galaxies.

In either case, the near future promises to be exciting for GRB astronomy as well as for studies of the high-redshift universe.

Acknowledgement. I am indebted to my collaborators for the various calculations mentioned in this overview: Rennan Barkana, Volker Bromm, Benedetta Ciardi, Steve Furlanetto, Jim Peebles, and Stuart Wyithe. This work was supported in part by NSF grants AST-0204514, AST-0071019, NASA grant ATP02-0004-0093, NATO grant PST.CLG.979414, and the John Simon Guggenheim Memorial Fellowship.

\section{References}

1. T. Abel, G.L. Bryan, M.L. Norman: Science 295, 93 (2002)

2. M.I. Andersen et al. : Astron. Astrophys. 364, L54 (2000)

3. R. Barkana, A. Loeb: Phys. Rep. 349, 125 (2001)

4. R. Barkana, A. Loeb: Nature 421, 341 (2003)

5. R. Barkana, A. Loeb: astro-ph 0305470 (2003)

6. A. W. Blain, P. Natarajan: Mon. Not. R. Astron. Soc. 312, L35 (2000)

7. J.S. Bloom, S.R. Kulkarni, S.G. Djorgovski: Astron. J. 123, 1111 (2002)

8. V. Bromm, P.S. Coppi, R.B. Larson: Astrophys. J. 564, 23 (2002)

9. V. Bromm, A. Loeb: Astrophys. J. 575, 111 (2002)

10. C.L. Carilli, N.Y. Gnedin, F. Owen: Astrophys. J. 577, 22 (2002)

11. R. Cen: astro-ph 0210473 (2002)

12. R. Cen: astro-ph 0303236 (2003)

13. B. Ciardi, A. Loeb: Astrophys. J. 540, 687 (2000)

14. B. Ciardi, A. Ferrara, S.D. M. White: astro-ph 0302451 (2003)

15. M. Dietrich, F. Hamann, I. Appenzeller, M. Vestergaard: astro-ph 0306584 (2003)

16. S.R. Furlanetto, A. Loeb: Astrophys. J. 579, 1 (2002)

17. S.R. Furlanetto, A. Loeb: Astrophys. J. 588, 18 (2003)

18. J.P.U. Fynbo et al. : astro-ph 0306403 (2003) 
19. J.E. Gunn, B.A. Peterson: Astrophys. J. 142, 1633 (1965)

20. Z. Haiman, G. Holder: astro-ph 0302403 (2003)

21. F. Hamann, M. Dietrich, B. Sabra, C. Warner: In: Origin and Evolution of the Elements, eds. A. McWilliam, M. Rauch (Proc. Carnegie Observ. Astrophys. Series, Vol. 4, 2003); astro-ph 0306068 (2003)

22. A. Heger, C.L. Fryer, S.E. Woosley, N. Langer, D.H. Hartmann: Astrophys. J. 591, 288 (2003)

23. S.R. Kulkarni et al. : SPIE 4005, 9 (2000)

24. D.Q. Lamb, D.E. Reichart: Astrophys. J. 536, 1 (2000)

25. A. Loeb, R. Barkana: Ann. Rev. Astron. Astrophys. 39, 19 (2001)

26. A. Loeb, P.J.E. Peebles: Astrophys. J. 589, 29 (2003)

27. J. Miralda-Escudé: Astrophys. J. 501, 15 (1998)

28. R. Perna, A. Loeb: Astrophys. J. 501, 467 (1998)

29. R. Somerville, M. Livio: astro-ph 030301 (2003)

30. D.N. Spergel et al. : astro-ph 0302209 (2003)

31. K.Z. Stanek et al. : Astrophys. J. Lett. 591, L17 (2003)

32. T. Totani: Astrophys. J. Lett. 486, L71 (1997)

33. R.A.M.J. Wijers, J.S. Bloom, J.S. Bagla, P. Natarajan: Mon. Not. R. Astron. Soc. 294, L13 (1998)

34. J.S.B. Wyithe, A. Loeb: Astrophys. J. 581, 886 (2002)

35. J.S.B. Wyithe, A. Loeb: Astrophys. J. 586, 693 (2003)

36. J.S.B. Wyithe, A. Loeb: Astrophys. J. Lett. 588, L69 (2003) 


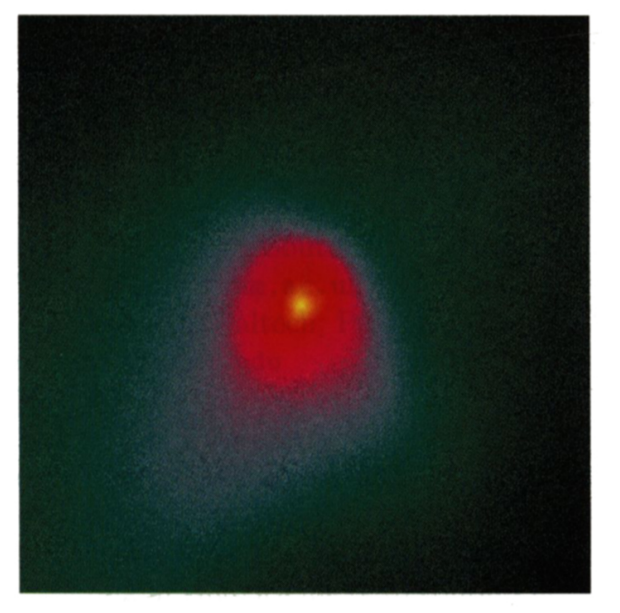
a) The coarse-grained morphology in a box with a linear physical size of
23.5 pc. At this time.
a gravitationally-bound clump has formed with a mass of $-1000 \mathrm{M}_{\odot}$

b) The fine-grained morphology in a box with a linear physical size of $0.5 \mathrm{pc}$. The central density peak accretes mass vigorously, and is accompanied by a secondary clump.

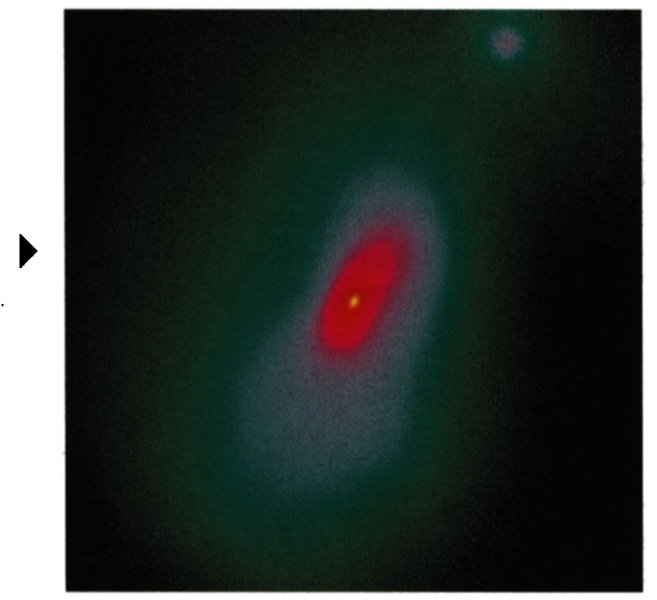

Numerical simulation of the collapse and fragmentation of a primordial cloud in 3D. We show the projected gas density at a redshift $\mathrm{z} \sim 21.5$, shortly after gravitational runaway collapse has converged at the center of the cloud. (From Bromm,V., \& Loeb, A., New Astronomy, 9, 353-364 (2004); astro-ph/0312456)

Plate (Loeb)

\section{Plate 6.}

